\title{
GESTIÓN DE LA INNOVACIÓN DIRIGIDA A LA CONSTRUCCIÓN DE TEJIDO SOCIAL Y PRODUCTIVO
}

\author{
Fernelly Ruiz Gómez
}

\section{Resumen}

La innovación entendida como un proceso social e interactivo que se fortalece en razón de la riqueza y densidad de las interacciones entre las personas y entidades involucradas en dicho proceso, en el presente artículo se propone que la gestión de la innovación debe estar orientada, fundamentalmente, a la construcción de redes de interacciones en pro de la innovación, con miras a construir tejido social y productivo ${ }^{2}$.

Este argumento se desarrolla mediante cinco puntos. Se inicia resaltando la importancia y el rol de las interacciones en la gestión de la innovación. Posteriormente se muestra que tanto la gestión del conocimiento como la gestión de la innovación no se pueden desarrollar satisfactoriamente sin un buen manejo de las interacciones entre agentes socioeconómicos. Luego se tratan dos herramientas para gestionar la innovación, los Sistemas de

1 Actual Director de la Escuela de Egresados del ITM. Ingeniero Electricista, Especialista en Telemática y candidato a Doctor en Estudios sobre ciencia y tecnología, y Gestión de la innovación tecnológica, Universidad del País Vasco.

E-mail: fernellyruiz@itm.edu.co

2 En ese sentido la reciente versión del Manual de Oslo (OECD, 2005) propone esta clasificación para las vinculaciones (linkages):

- "Open information sources: openly available information that does not require the purchase of technology or intellectual property rights, or interaction with the source.

- Acquisition of knowledge and technology: purchases of external knowledge and/or knowledge and technology embodied in capital goods (machinery, equipment, software) and services, which do not involve interaction with the source.

- Innovation co-operations: active co-operations with other enterprises or public research institutions for innovation activities (which may include purchases of knowledge and technology)" (OECD, 2005, p. 82). 
Innovación y las Redes de Innovación, las cuales permiten a los gestores estudiar los contextos de su interés y orientar la capacidad innovativa hacia fines deseados.

A continuación se propone a los gestores un modelo que vincula la innovación, el conocimiento y el trabajo en red, y finalmente, se argumenta que también se requiere el conocimiento sobre el contexto que la aceptará o rechazará, para poder aumentar la probabilidad de éxito de la iniciativa innovadora.

El presente escrito es un análisis crítico sobre la gestión de la innovación, desprendido del trabajo investigativo adelantado en el programa de Doctorado en Estudios sobre ciencia y tecnología, y Gestión de la innovación tecnológica, servido por la Universidad del País Vasco.

\section{Palabras clave}

Innovación, Gestión de la innovación, Interacciones y trabajo en red (Networking).

\section{Abstract}

The innovation is conceived as a social and interactive process, which is strengthens in reason of the wealth and density of the interactions among people and entities involved in this process. This article proposes that the innovation management should be guided fundamentally in order to construct networks in pro of the innovation and to construct social and productive interactions.

This argument is developed by means of five points. It begins mentioning the importance and functions of the interactions in the innovation management. Later it is shown that too the knowledge management and the innovation management cannot be developed satisfactorily without a good management of the interactions among socioeconomic agents.

Then two tools are treated in order to manage the innovation, the Innovation Systems and the Innovation Networks, both permit to managers to study the contexts of their interest and to guide the innovative capacity toward wanted goals. 
Next it suggest to managers a model that links the innovation, the knowledge and the networking, and finally, before passing to the conclusions, it argues that is also necessary to know the context that will accept or will reject the innovations, in order to increase their probability of success.

This article is a critical analysis originated by the research work developed in the PHD program Science and technology studies and, Technological innovation management, served by the University of the Basque Country.

\section{W}

Innovation, Innovation management, Interactions and Networking. 



\section{WNTRODLCCÓN}

La innovación es un proceso social e interactivo basado en el conocimiento, del cual se desprenden ideas, procesos, productos o servicios novedosos, con los cuales se pretenden satisfacer las necesidades o expectativas de la sociedad que se sirve de ellos. Este proceso es impulsado, en primera instancia, por la tendencia intrínseca del ser humano hacia el aprendizaje y la creación de nuevas cosas, y en segunda instancia, por la necesidad empresarial de competir entre sí, buscando una mayor aceptación y consumo de sus productos o servicios (Lundvall, 1992; Nelson, 1993; Olazarán y Uranga, eds., 2000, Pavitt, 2003).

Concebirla como un proceso social e interactivo implica considerar que la innovación se fortalecerá en razón de la riqueza y densidad de las interacciones entre las personas y entidades involucradas en dicho proceso. Ahora, dado el rol preponderante de la innovación en la sociedad moderna, (Pavitt, 2003) ha sido necesario desarrollar un proceso complementario denominado gestión de la innovación, útil para implementar contextos que potencialicen las capacidades creativas de las personas y los equipos de trabajo. Dicha gestión es desarrollada normalmente desde los gobiernos y las mismas empresas, es decir, que se soporta en políticas públicas y en estrategias empresariales, pero en el fondo, es una tarea de la sociedad en su conjunto, razón por la que debe soportarse también en redes de trabajo empresariales, institucionales y políticas.

Por tanto, la gestión de la innovación es también un proceso clave, no solo para elevar la competitividad empresarial, sino también para canalizar y focalizar el potencial humano de una región, con base en sus características y fortalezas idiosincrásicas.

En ese orden de ideas, en el presente artículo se considera que la gestión de la innovación debe estar orientada fundamentalmente a la construcción de redes de interacciones en pro de la innovación. Se trata entonces, de conferir suficiente importancia a las relaciones o interacciones entre los agentes socioeconómicos, con miras a construir tejido social y productivo. 


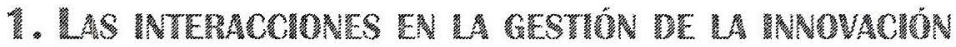

¿Por qué son importantes las interacciones para la gestión de la innovación? Y, ¿qué papel juegan las interacciones en la gestión de la innovación?

Los sistemas productivos son abiertos y dinámicos como consecuencia de las interacciones que se producen entre los diversos agentes socioeconómicos que lo constituyen y circundan, por tanto, inevitablemente la gestión de la innovación estará profundamente emparentada con dichas interacciones $\mathrm{y}$, consecuentemente, el fomento de las interacciones es equivalente a la construcción de redes de tejido social y productivo.

Luego, con la gestión de la innovación lo que se pretende es intervenir una realidad, ejercicio que debe partir de la identificación de los espacios de interacción cultural, es decir, debe comenzar por caracterizar y esquematizar una asociación de prácticas que identifican un espacio común de interacción social.

Para lograrlo, es fundamental definir al menos dos aspectos determinantes: primero, cuál es el elemento aglutinante, el que determinará cuál es el espacio cultural, por ejemplo: una región geográfica, una dinámica económica, un sector estratégico para el desarrollo, un sector en conflicto, etc., y segundo, debe expresar conceptualmente los elementos característicos de dicho espacio ${ }^{3}$.

3 En el caso de Colombia, no existe una definición ni organización institucional ni de ninguna otra clase, del concepto de región; la realidad jurídica y política está recubierta por aquellas entidades territoriales que son los Departamentos, los cuales gozan de un fuerte arraigo histórico. Pero a pesar de algunos esfuerzos por reordenar el territorio a partir del concepto de región funcional que viabilice un mejor equilibrio territorial, estas iniciativas hasta hora se han visto frustradas por diversos intereses de la clase política del país.

En contraste, para los países más desarrollados el concepto hace referencia a una entidad operativa a nivel meso, en términos administrativos y políticos, que funciona entre los gobiernos locales y los nacionales. Así se definió en 1998 por el proyecto REGIS (Regional Innovation Systems) denominado "Designing for the future". Esta definición también incluye otras posibles formas de conformación de las regiones, por ejemplo, algunas veces estas son designadas desde arriba como una reorganización administrativa de los estados-nación, tal como sucedió en Francia 
Visto desde un punto de vista filosófico, específicamente de la Teoría combinatoria de las representaciones científicas (Ibarra y Mormann, 2000), un paso importante para los gestores es representar científicamente los espacios culturales que intervendrán, no como imágenes especulares o isomórficas del contexto que se estudia, sino como sistemas de representaciones en los que se combinan diferentes tipos de representaciones propias del espacio en cuestión.

Por lo tanto, una nueva mirada de la gestión de la innovación, centrada en las interacciones entre los agentes socioeconómicos, sugiere que la naturaleza de las representaciones sobre las que se basa el ejercicio sean no-isomórficas, y más bien, sean construidas con una cierta lógica suficientemente aceptable para la sociedad que se beneficiará del análisis, esto es, representaciones homológicas o functoriales, que no dependen exclusivamente de una semejanza desde el punto de vista de los objetos o desde el punto de vista de las estructuras.

En una representación lógica de este tipo, no son solamente los objetos (por ejemplo las innovaciones en productos) ni las estructuras (por ejemplo las innovaciones en procesos $u$ organizacionales) los que son considerados y representados, sino también las relaciones lógicas. Este enfoque induce un contextualismo u holismo local, de tal forma que se ofrezca a los interesados en conjunto una representación más rica que la que ofrecerían representaciones individuales, como tratando de producir un "imagen lógica" del contexto cultural en cuestión.

Se hace necesario entonces, utilizar estrategias para distinguir determinadas actividades singulares, para capturar determinadas marcas de la realidad, teniendo muy presente que las represen-

\footnotetext{
cuando las regiones fueron descentralizadas por el gobierno central. En otras ocasiones han sido creadas por presión desde abajo, como en el caso de Inglaterra donde la heterogeneidad cultural ha sido expresada en demandas para diferenciar las estructuras gubernamentales regionales. En otros casos la regionalización es una combinación de ambas, como en Italia o España. Otros casos son los sistemas federales con poderes equivalentes a las regiones (Gómez Uranga et al., 2002).
} 
taciones no están simplemente ahí, más bien se las construye con ciertos fines. Para lograr esa visión exitosa de la realidad propia de un determinado espacio cultural, el alcance del análisis debe estar determinado por la búsqueda de explicaciones suficientemente significativas para la sociedad de este espacio, ya que cada ámbito cultural tiene sus representaciones particulares, no semejantes a las de sus ámbitos vecinos; consecuentemente, la gestión de la innovación debe estar alimentada por explicaciones suficientemente representativas, por supuesto, no absolutas ni completas, de cómo operan los mecanismos de producción del conocimiento allí donde se aplica el análisis.

Así las cosas, el ejercicio debe focalizarse, más que en aspectos sustantivos de las empresas, en el estudio de la interacciones de los agentes socioeconómicos en los diferentes marcos culturales de un sector económico, una región o un país. Esto implica que se identifiquen y caractericen las interacciones que se presentan en la producción del conocimiento para un determinado marco cultural, de tal forma que se expliquen los mecanismos que generan las creencias, estabilizan las convicciones y sirven de pauta para la acción a los elementos diversos y heterogéneos que componen el escenario en cuestión.

En conclusión, las interacciones juegan un papel central en la gestión de la innovación, y su análisis facilita la elección de los contrastes y la mirada eco-sistémica que debe darse a la explicación para que satisfaga esos intereses. De hecho "los esfuerzos para el desarrollo de nuevos indicadores que ofrezcan una mejor representación de los desarrollos científico-tecnológicos y sus consecuencias sociales y naturales pueden ser baldíos si no ofrece una mejor comprensión de los mecanismos de producción del conocimiento y del desarrollo social, así como de la relación entre ambos". Es entender que la innovación es más que una articulación de capacidades y recursos, que incluye además un tejido de actitudes como parte del contexto de innovación" (Ibarra, 2003, págs. 2 y 8). 


\section{GESTIONAR El CONOCIMIENTO Y LA INNONACIÓN ES, DE CIERTA FORMA, GESTIONAR LAS INTERACCIONES}

La innovación es un proceso interactivo basado en el conocimiento, por lo tanto, un importante componente de la gestión de la innovación es la gestión del conocimiento. Además, ambos tipos de gestión, que tienen mucho en común, no se pueden desarrollar satisfactoriamente sin un buen manejo de las interacciones entre agentes socioeconómicos.

Keith Pavitt (2003) argumenta que del proceso innovativo se desprenden al menos tres subprocesos que se sobreponen entre sí: Producción de conocimiento; transformación de conocimiento en productos, servicios, procesos y sistemas y; adaptación frente a las necesidades y demandas futuras del mercado. Esto genera un panorama potencialmente confuso que impacta a la sociedad en tres niveles: cognitivo, organizacional y económico.

El "juego" consiste en la exploración y explotación de oportunidades para obtener productos, servicios, procesos y/o sistemas nuevos o mejorados, basándose también en una ventaja técnica ("Know how"), un cambio en la demanda del mercado o una combinación de ambas. Todo en un ambiente de incertidumbre que imposibilita predecir exactamente el costo y la construcción de un artefacto novedoso, así como las reacciones de los usuarios ante ello.

Así las cosas, quienes compiten y quienes innovan pasan inevitablemente por procesos de aprendizaje cruzados por experimentación (ensayo y error) y/o de mejor entendimiento (teoría). Luego la gestión del conocimiento se transforma en una interacción entre empresas y tecnología que tiene implicaciones como:

- Una especialización y profesionalización de la naturaleza del conocimiento en el cual se basan, razón por la que el futuro de las empresas está fuertemente condicionado por lo que han aprendido en el pasado.

- Las empresas especializadas en diferentes campos del conocimiento tienden a destacarse, dependiendo del campo en el que se desempeñan. 
- La gestión del conocimiento difiere significativamente entre grandes y pequeñas empresas, a pesar de que la relación entre las grandes corporaciones y las Pymes se está incrementando de forma importante mediante una desintegración vertical de la producción.

En relación con la especialización del conocimiento empresarial, se ha generado una tendencia hacia el desarrollo de laboratorios de $\mathrm{I}+\mathrm{D}$ en las grandes empresas, dedicados fundamentalmente a la producción de conocimiento para la explotación comercial; además, el surgimiento de una innumerable cantidad de pequeñas empresas que proveen mejoramientos continuos en la producción de bienes especializados.

A este intrincado panorama se suma una combinación de conocimiento de origen privado aplicado en los negocios de las empresas y de conocimiento desarrollado en el sector público, generalmente diseminado por universidades e instituciones similares. Estas formas de especialización del conocimiento se presentan simultáneamente en un patrón de cambio técnico heterogéneo, dependiente de la trayectoria histórica, científica y tecnológica, que resalta el matiz interactivo de la innovación y que, por supuesto, es bastante dificil de gestionar.

De otro lado, el amplio rango de campos del conocimiento que contribuyen al diseño y construcción de cada producto incrementa la complejidad de los artefactos, generándose otra tendencia, la modularización e integración en los sistemas productivos. De ahí la importancia de las arquitecturas modulares en los productos, donde los componentes de interfaz son estandarizados e interdependientes, obligando a los nodos productivos de un sistema a transferirse información codificada trabajando juntos (interactuado) en relación con el mercado.

Esta complejidad modular no se supera solamente codificando información, se hace necesaria una superposición entre las empresas y sus correspondientes competencias para facilitar la transferencia de conocimiento tácito, con las consecuencias y los 
desbalances a que haya lugar, incrementándose así las dificultades en el diseño e integración entre sistemas productivos.

Consecuentemente, uno de los mayores retos de la gestión de la innovación en las empresas exitosas es hacer corresponder productos, procesos, sistemas, servicios y prácticas organizacionales, con las demandas del mercado y la demanda potencial. Esto es, los más importantes procesos a gestionar en materia de innovación son aquellos para responder a las necesidades y demandas del mercado y así, hacer corresponder las prácticas organizacionales con las oportunidades tecnológicas; asunto que implica enfrentarse las consecuencias negativas de la especialización del conocimiento, así como a cambios que implican rupturas con los paradigmas prevalecientes en las empresas.

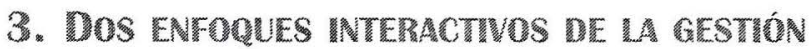

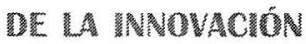

Debido a la dinámica tecnológica y económica moderna, la intensidad y características de las interacciones entre los agentes de un sistema productivo permanecen en continuo desequilibrio. Esto obliga a los gestores a desarrollar herramientas analíticas y de gestión que les permitan captar los rasgos más significativos de dicha dinámica, así como orientar la capacidad innovativa hacia fines deseados.

Las relaciones y conexiones entre actividades (productiva, comercial, técnica, financiera y asistencial), actores (empresas industriales del sistema productivo local) y recursos (humanos, naturales, infraestructuras) han sido crecientes durante un largo período de tiempo, por lo que dependen del estilo de vida sociocultural y productivo de los territorios (Vázquez, 1999).

Pero la forma como se circunscribe la innovación a la esfera empresarial y su débil vinculación con la sociedad que la convalida es realmente un problema. Aunque se le confiere importancia a las vinculaciones empresariales, el análisis es todavía poco profundo e impreciso, lo que acarrea consecuencias bastante negativas a la 
hora de desprender análisis confiables y dicientes para quienes pretenden gestionar la innovación.

Esta dificultad ha obligado al desarrollo de herramientas que resaltan la importancia de las interacciones para dicha gestión.

\section{3. 售 Las sistemas te innovacton}

La reciente versión del Manual de Oslo (OECD, 2005) anota que, si se ve el proceso de innovación sistemáticamente, las mediciones pueden proveer información útil, por ejemplo, sobre los vínculos de las firmas con otros actores o sobre la forma como protegen sus innovaciones; pero, asegura también, hay limitaciones ya que generalmente los datos recolectados requieren ser combinados con otros, que a menudo no están disponibles o no son especificados en la memoria empresarial.

Para facilitar el estudio y la gestión de la innovación se ha desarrollado una herramienta muy utilizada hoy en día, los Sistemas de Innovación (Lundvall ed., 1992; Nelson y Rosenberg, 1993; Freeman, 1995; Edquist ed., 1997, entre otros). Estos son un marco de análisis mediante el cual se pretende conocer la estructura económica y social de un determinado territorio, fundamentándose, en gran medida, en la teoría del aprendizaje interactivo la cual se centra en las relaciones existentes entre los diversos agentes que constituyen un Sistema de Innovación en particular.

El enfoque de Sistema de Innovación pretende analizar la existencia e interacción de los agentes presentes en un determinado territorio (nación o estado, región, etc.) tales como instituciones gubernamentales, clusters, universidades, industrias, etc., además de conocer las competencias que éstos poseen, a fin de aportar a las autoridades (nacionales, regionales o locales) elementos de juicio que les faciliten la gestión de la innovación (Lundvall ed., 1992).

Por eso es fácil encontrar una amplia gama de proyectos de investigación en los que se estudian las relaciones que se establecen entre los agentes de los Sistemas de Innovación (European Planning Studies, Vol. 8, Not. 4, 2000). Además, se han creado diferentes modelos de simulación para tratar de medir las carac- 
terísticas de dichos Sistemas en diferentes entornos (Simulating Self-Organizing Innovation Networks" -SEIN) ${ }^{4}$

De hecho, es posible diferenciar tres líneas de actuación en las que se han desarrollado los Sistemas de Innovación (Olazarán y Uranga, eds., 2000, p. 13-18):

- Estudios basados en Políticas de Innovación, comparando las características de diferentes Sistemas de Innovación por medio de análisis de Benchmarking.

- Estudios que pretenden formalizar el concepto de los Sistemas Nacionales de Innovación a través de modelos descriptivos o analíticos.

- Estudios sobre los Sistemas Nacionales y/o Regionales de Innovación de determinados países y/o regiones.

Sin embargo, aún hay un amplio campo por abordar en estos Sistemas, pudiéndose al menos citar tres posibles áreas objeto de estudio:

- En primer lugar, se requiere de una relación más explícita entre el concepto de Sistemas Nacionales de Innovación y el crecimiento económico.

- En segundo lugar, la relación existente entre el Sistema de Innovación de un determinado país y el resto de los subsistemas (mercado de trabajo, sistemas financieros, etc.) está

4 De estos estudios y modelos propuestos se han soportado en diversas definiciones sobre el concepto de los Sistemas de Innovación, por ejemplo:

"network of institutions in the public and private sectors whose activities and interactions initiate, import, modify and diffuse new technologies" (Freeman, 1987).

"a number of elements and the relationships between these elements... which interact in the production, diffusion and use of new, and economically useful knowledge..." (Lundvall ed., 1992).

"The National Systems of Innovation are constituted by "interconnected agents" that interact influencing on the execution of the innovation in the national economy. These interactions occur into a specific context and under certain shared norms, routines and established practices." (Nelson y Rosenberg, 1993). 
lejos de ser abordada de modo exhaustivo. Esta limitación es aún más relevante, al tratarse de sistemas abiertos, en los que su fortaleza depende entre otros aspectos, de su relación con varios sub-segmentos de la economía.

- Por último, debe mencionarse el limitado conocimiento que se dispone de las propiedades dinámicas en los Sistemas de Innovación, especialmente en lo que concierne a su estabilidad y evolución estructural.

Por eso, aunque esta herramienta analítica facilita significativamente la gestión de la innovación, es aún un problema investigativo, por cuanto las características propias de cada región y de cada sector económico son un universo por descubrir.

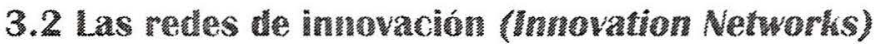

Como ya se ha dicho, en torno a la innovación los agentes socioeconómicos interactúan para fomentar, desarrollar e intercambiar diversos tipos de conocimiento, información, experiencias y otro tipo de recursos.

En muchos casos, esta actividad innovadora se desarrolla basada en las competencias presentes en un territorio (Olazarán y Uranga eds., 2000, p.16), sin embargo, debido al cambio que se está produciendo en el entorno socio-económico global, las interacciones entre diferentes regiones y países se están convirtiendo en algo cotidiano, por lo tanto, la introducción de nuevos agentes y la generación de nuevos tipos de interacciones son una de las características más marcadas hoy día en la dinámica de los sistemas, asunto que ha llevado a la necesidad de hablar de redes de interacción al interior y entre los sistemas, algo que se está denotando como Innovation Networks o Redes de Innovación.

El tratamiento de este fenómeno es relativamente reciente ya que emergió a comienzos de los 90 y es considerado como una herramienta útil para explicar algunos fenómenos tales como las dinámicas de las organizaciones empresariales y las de las industrias de un sistema productivo local (Vázquez, 1999). 
Por ejemplo, de las redes de relaciones entre estado-empresauniversidad, lo que se ha denominado la Triple Hélice, puede surgir un nuevo régimen global, de tal forma que la red resurge como una nueva unidad de evolución. Esto significa que cuando una estructura en forma de red haya adquirido cierta estabilidad, podrá co-evolucionar con el sistema en su conjunto. Parece claro entonces que la generación de redes de interacción entre los agentes de un Sistema de Innovación es uno de los principales argumentos por los que éste evoluciona y se desarrolla. En este contexto, las redes representan un mecanismo para la difusión de innovaciones a través de colaboraciones y de relaciones interactivas, es decir, como una expresión de la gestión de la innovación.

Por lo tanto, debido al papel que desempeñan las redes en un Sistema de Innovación eficiente, los lazos de unión entre sus agentes deben ser muy estrechos, ya que los procesos de generación y difusión de nueva tecnología descansan en cierto modo sobre la reducción de los costes de transacción a través de las redes. Así mismo, una Red de Innovación bien establecida puede contener una gran inercia y una gran dependencia de su trayectoria histórica y tecnológica (Carlsson y Jacobson, 1997; Archibugi, Howells y Michie eds., 1999).

En ese orden de ideas, las Redes de Innovación son un concepto poliédrico, dependiente de la perspectiva que el investigador adopte. Es por esto que la literatura filosófica, económica, sociológica, política, entre otras, ha comenzado a demostrar que los recientes desarrollos en la generación de nuevo conocimiento pueden ser conceptualizados en términos de Redes de Innovación o Innovation Networks. Pero, a pesar de esto, aún hay un largo camino por recorrer debido a la gran variedad de perspectivas que éstas permiten adoptar, asunto que ha inducido a la comunidad científica a desarrollar modelos de simulación que permiten determinar los posibles eventos que pudieran ocurrir en las redes y en los Sistemas de Innovación a los que pertenecen, para que los investigadores puedan considerar diferentes escenarios en el desarrollo de un Sistema. 
Por tanto, la heterogeneidad y naturaleza contingente de la innovación, hace que los gestores corporativos no se puedan basar solamente en buenas prácticas de gestión de la innovación, y que deban aceptar que la producción de conocimiento, su distribución, así como el desarrollo social y económico son procesos complejos y directamente relacionados con las interacciones y dinámicas propias de las redes o sistemas productivos y sociales en que se hayan inmersas.

\section{4. ¿CÓMO GESTIONAR LA INNOVACIÓN CENTRÁNDOSE EN EL TRABAJO EN Pres?}

Actualmente el estudio del trabajo en red, es decir, de las interacciones entre los agentes socio-económicos, presenta serias dificultades, ya que la mayor parte de los indicadores de innovación no contribuyen de un modo directo a conocer la dinámica de estas, además, las medidas empleadas realmente no se refieran a interacciones, se trata de medidas que conciernen a los agentes que constituyen los Sistemas de Innovación.

De hecho, para identificar y caracterizar las interacciones, o las posibles redes de innovación, presentes en un sistema, y comprenderlas a fondo, se debe llegar hasta un análisis de segundo y tercer orden que permita "elevar" la mirada para verlas desde "arriba", desde una mejor perspectiva, y no simplemente ubicándose en la empresa para tratar de observar sus vinculaciones.

Expresado de otra forma, cuando se observa una red desde uno de sus nodos, la mirada es limitada y no se alcanzan a ver todos los detalles de la misma, en cambio, cuando se mira desde un plano elevado, que permita una vista panorámica de sus nodos y la naturaleza de sus interacciones, se obtiene más y mejor información sobre ella.

Un análisis de esta naturaleza exigiría varios acercamientos a los nodos para caracterizar una red. Un primer acercamiento sería para determinar su existencia y naturaleza, y luego, serían necesarios otros acercamientos, en cada uno de los cuales, se elevaría el 
nivel de análisis a un segundo y tercer orden, para llegar a un punto que ofrezcan una mejor perspectiva "visual" de la red y, así, poder intervenirla mejor, sea para fortalecerla, ampliarla o dinamizarla, entre otros posibles fines de la gestión de la innovación.

Una buena gestión de la innovación debe reconocer la complejidad tanto de la innovación como del Networking (trabajo en red o establecimiento de redes), es decir, debe considerar que la innovación se desarrolla recíproca y sistemáticamente mediante la creación y el mantenimiento de redes.

Ahora, se debe mirar el trabajo en red como un proceso, superando las miradas estructurales convencionales como las planteadas por Grandori \& Soda (1995, p. 184), quienes definen las redes como un modo de organización de las actividades económicas a través de co-ordinación inter-firmas.

Se sugiere entonces, asumir posturas como las de Nahapiet \& Ghoshal (1998), quienes resaltan la importancia del capital social y consideran que el desarrollo del proceso innovativo debe ser intervenido mediante la creación y el mantenimiento en el tiempo de redes sociales, destacando a su vez, la necesidad de reconocer sus cualidades emergentes y formativas, es decir, confiriendo suficiente importancias a algunas actividades frágiles y exploratorias basadas en contactos embrionarios y relaciones medio formadas.

La visión de proceso sugerida permite analizar la forma como el proceso de conformación de redes moldea el conocimiento, la información y la innovación que se difunden. Por tanto, la gestión de la innovación debe resaltar la importancia central de los "encuentros accidentales" para la innovación, particularmente en las fases nacientes en la formación de redes, caracterizadas por las relaciones interpersonales, informales y oportunistas.

En este contexto, la innovación es conceptualizada como una serie de episodios de transformación particular de conocimiento e información, en los cuales, a la vez que el conocimiento y la información se esparcen, son transformados por los actores sociales de la red (Nonaka et al., 2003; Swan et al., 2003). 
En la siguiente figura se presenta un modelo que armoniza con los puntos teóricos mencionados y su relación con los diferentes episodios del proceso innovativo (Swan et al., 2003, p. 685).

El modelo se focaliza en la interrelación entre las tres dimensiones críticas identificadas hasta ahora: actividad de trabajo en red (Networking), los atributos del conocimiento y los episodios del proceso de innovación. Como puede observarse, la figura sugiere que las formas dominantes de difusión, utilización y generación de conocimiento pueden variar de un episodio a otro, por tanto, la dirección y el ámbito del proceso innovativo deberá reflejar estas co-dependencias.

UN MOdELo PARA RELACIONAR EL TRABAjo EN RED (NETWORKING), EL CONOCIMIENTO Y LA INNOVACIÓN ${ }^{5}$

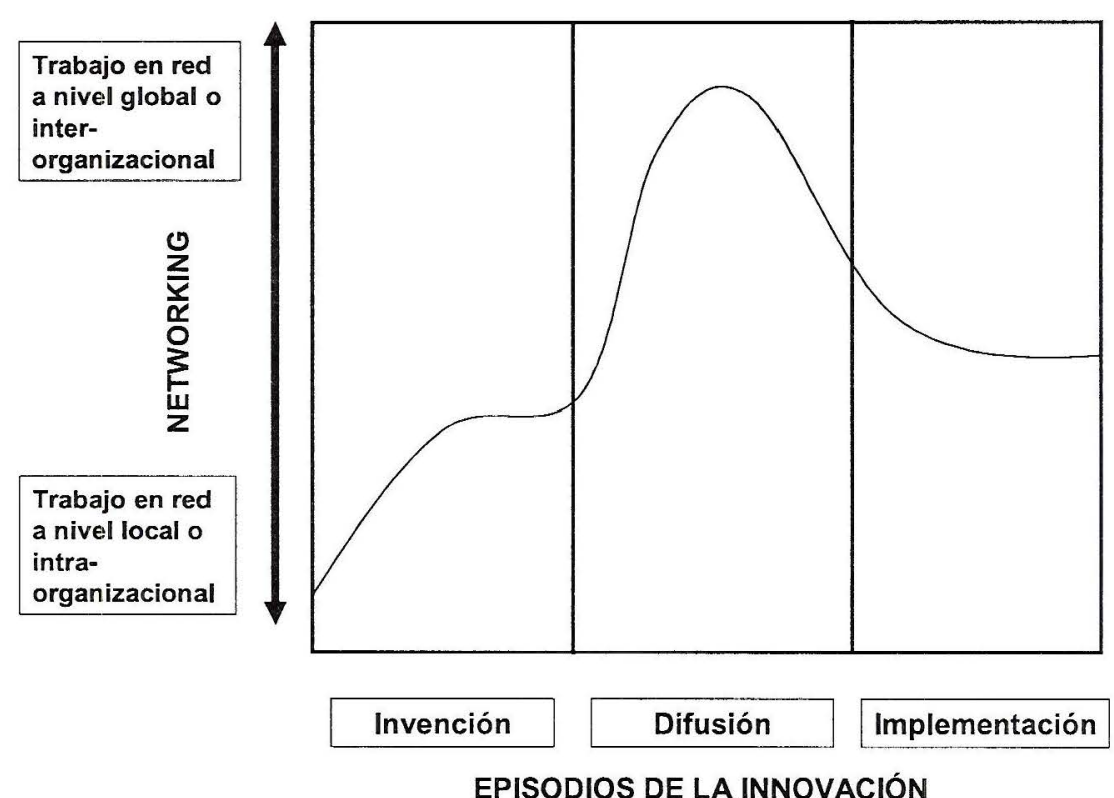

EPISODIOS DE LA INNOVACIÓN

$5 \quad$ Fuente: Swan et al., 2003, p. 685. 
A continuación se caracterizan los elementos constitutivos y los supuestos asumidos por este modelo.

Invención: Durante este episodio de la innovación la atención se centra en la construcción social y creación de nuevo conocimiento a través de un proceso exploratorio y altamente personalizado, entre un débil y poco definido grupo social que cuenta con conocimiento tácito relevante y algunos intereses comunes. En la figura esto es descrito en la zona donde se presenta un trabajo en red local o intra-organizacional.

Durante este episodio el trabajo en red es informal e interpersonal, con el fin de identificar potenciales participantes que poseen información y experiencia que puede ser relevante para el desarrollo de nuevos productos o servicios.

El papel clave de las coaliciones sociales y los equipos se dirige al chequeo e interpretación del conocimiento, por tanto, el énfasis aquí es compartir y crear conocimiento, más que intercambiar información o artefactos.

Difusión: Durante el episodio de difusión el énfasis recae sobre la codificación y comunicación del conocimiento por medio de unas redes más globales e inter-organizacionales. Las ideas ahora han sido cristalizadas como nuevos artefactos técnicos, productos o servicios. El principal rol del trabajo en red es diseminar conocimiento para legitimar inventos particulares o nuevas ideas, a fin de que sean aceptadas y adoptadas por una amplia comunidad.

La difusión involucra un proceso social de intercambio (formal e informal) de información entre miembros de un sistema social. Este proceso es desigual y, en muchos casos, conflictivo.

Implementación: Este episodio se refiere a la apropiación local de nuevas ideas como soluciones organizacionales específicas. A menudo las innovaciones no pueden ser adoptadas por las organizaciones de manera directa o en la forma original. Por tanto requieren modificaciones y reconfiguraciones para adoptarlas a contextos técnicos y organizacionales específicos. En este episodio se presenta una de-construcción y re-construcción de conocimiento. Durante la implementación tiende a converger el trabajo intra e inter organizacional. 
Por lo tanto, el gestor de la innovación debe ser consciente del comportamiento y características del Networking entre los agentes socioeconómicos involucrados en cada episodio, a fin de poder facilitar el desarrollo del proceso innovativo.

\section{LA GESTÓN DE LA INNOVACIÓN REQUUERE COMPRENDER LA

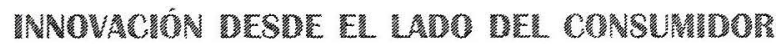

Es común que se gestione la innovación desde las empresas y las instituciones, pero ¿qué decir si se mira del lado del consumidor? ¿Será importante evaluar la forma como reaccionan las personas de un marco cultural dado ante las ideas, productos o servicios nuevos?

Como ya se dijo, si se confiere la importancia que merecen las relaciones o interacciones entre los agentes socioeconómicos (Networking), con miras a construir tejido social y productivo, debe entenderse que el significado de innovación no existe en sí mismo, sino que depende de su contexto de producción, aquel en el que se contrasta y valida.

Es necesario entonces, también evaluar la innovatividad (innovativeness) entendida como las diferencias entre individuos en la forma como reaccionan ante las innovaciones o las nuevas cosas (Goldsmith y Foxall, 2003). Es decir, observar la innovatividad es observar la innovación desde el lado del consumidor.

Este concepto se fundamenta en la teoría de la difusión de la innovación, la cual describe la forma como las cosas nuevas (por ejemplo un nuevo producto) se difunden y son aceptadas a través de un sistema social (Rogers, 1995). Esta teoría ha sido, y aún permanece, como un tópico importante para la gerencia del mercadeo y el estudio del comportamiento de los consumidores por parte de las empresas.

Existen al menos tres aproximaciones al concepto de innovatividad, cada uno de los cuales conlleva sus propias implicaciones a la hora de medirla (Goldsmith y Gordon, 2003., p. 324-325).

Basada en la conducta personal: Esta perspectiva de innovatividad identifica el concepto con el acto de adopción de una innovación. 
Los consumidores son clasificados como afines a las innovaciones o no, dependiendo de la facilidad con que adoptan un nuevo producto o lo rechazan. De hecho el grado de innovatividad que poseen depende de lo rápido que adoptan una innovación luego de que la han encontrado.

Personalidad global característica: esta visión argumenta que la innovatividad es un tipo de marca personal. Los rasgos personales son vistos como unos patrones relativamente duraderos de comportamiento o cognición que diferencian una persona de otra. La innovatividad describe el rango de reacciones desde una actitud positiva hacia el cambio hasta una actitud muy negativa.

Personalidad característica en un dominio específico: los consumidores son vistos con más o menos innovatividad en relación con categorías de productos específicos, por ejemplo, entusiasmo por la moda, un conocedor de vinos, o un aficionado al cine.

Por lo tanto, aunque la gestión de la innovación actúa básicamente sobre aquellos que desarrollan una innovación, por ejemplo, las empresas y/o las instituciones, requiere conocimiento sobre el contexto que la aceptará o rechazará, para poder aumentar la probabilidad de éxito de la iniciativa innovadora.

\section{CONCLUSIONES}

La gestión de la innovación es un proceso clave con el que se busca fortalecer la innovación, no solo para elevar la competitividad empresarial, sino también para canalizar y focalizar el potencial humano de una región, con base en sus características y fortalezas idiosincrásicas.

Para intervenir con más acierto una realidad, mediante esta gestión, el ejercicio debe focalizarse, más que en aspectos sustantivos de las empresas, en el estudio de la interacciones de los agentes socioeconómicos en los diferentes marcos culturales de una región o un país. Por lo tanto, las interacciones juegan un papel central en la gestión de la innovación, ya que facilita la elección de los contrastes y la mirada eco-sistémica que debe darse a la explicación para que satisfaga esos intereses. 
Pero, debido a la dinámica tecnológica y económica moderna, la intensidad y características de las interacciones entre los agentes de un sistema productivo permanecen en continuo desequilibrio. Esto obliga a los gestores a desarrollar herramientas analíticas y de gestión que les permitan captar los rasgos más significativos de dicha dinámica, así como orientar la capacidad innovativa hacia fines deseados.

Una de ellas son los Sistemas de Innovación, con lo que se pretende analizar la existencia e interacción de los agentes presentes en un determinado territorio (nación o estado, región, etc.), tales como instituciones gubernamentales, clusters, universidades, industrias, etc., además de conocer las competencias que éstos poseen, a fin de aportar a las autoridades (nacionales, regionales o locales) elementos de juicio que les faciliten la gestión de la innovación.

Dado que la introducción de nuevos agentes y la generación de nuevos tipos de interacciones son una de las características más marcadas hoy día en la dinámica de los sistemas, la generación de redes de interacción entre los agentes de un Sistema de Innovación es uno de los principales argumentos por los que éste evoluciona y se desarrolla.

Este comportamiento ha desembocado en un nuevo concepto, algo que se está denotando como Innovation Networks o Redes de Innovación. En este contexto, las redes representan un mecanismo para la difusión de innovaciones a través de colaboraciones y de relaciones interactivas, es decir, como una expresión de la gestión de la innovación.

Se hace necesario entonces que los gestores corporativos acepten que la producción de conocimiento, su distribución, así como el desarrollo social y económico son procesos complejos y directamente relacionados con las interacciones y dinámicas propias de las redes o sistemas productivos y sociales en que se hayan inmersas.

Por lo tanto, una buena gestión de la innovación debe reconocer la complejidad tanto de la innovación como del Networking (trabajo 
en red o establecimiento de redes), es decir, debe considerar que la innovación se desarrolla recíproca y sistemáticamente mediante la creación y el mantenimiento de redes.

\section{BUELOCRAFIA}

ARCHIBUGUI, D., HOWELLS, J. y MICHIE, J. (eds.) (1999). Innovation Policy in a Global Economy. Cambridge: Cambridge University Press.

CARLSSON, B. y JACOBSON, S. (1997). Diversity creation and technological systems: A technology policy perspective. En: Edquist, C. (ed.), Systems of innovation: Technologies, institutions and organizations. Londres: Pinter Publishers.

EDQUIST, C. (ed.) (1997). Systems of innovation: Technologies, institutions and organizations. Londres: Pinter Publishers.

European Planning Studies (2000). Vol. 8, Not. 4.

FREEMAN, C., 1987. Technology Policy and Economic Performance: Lessons from Japan. London: Pinter.

FREEMAN, C. (1995). The National System of Innovation in historical perspective. En: Journal of Economics. Cambridge.

GOLDSMITH, R. y FOXALL, G. (2003). The Measurement of Innovativeness. En: L. V. Shavinina (Ed.), International Handbook on Innovation. Oxford: Elsevier Science.

GÓMEZ, M., DIEZ, M. Jubero, y ETXEBARRIA, G. (2002). Integrating Technological and social aspects of foresight in Europe, State of the Art Report on Regional Development. Applied Economics I. Bilbao: Universidad del País Vasco.

GRANDORI, A. y SODA, G. (1995). Inter-firm networks: Antecedents, mechanisms and forms. Organization Studies, 16, 184-214.

IBARRA, A. y MORMANN, T. (2000). Una teoría combinatoria de las representaciones científicas. En: Crítica. Vol. 32. No. 95. México: UNAM.

IBARRA, A. (2003). ¿Son genuinas las representaciones científicas? En: Minhot, L. y Testa, A. Representación en Ciencia y Arte. Córdoba: Brujas: Universidad Nacional de Córdoba.

LUNDVALL, B. A. (ed.) (1992). National Systems of Innovation: Towards a Theory of Innovation and Interactive Learning. Londres: Pinter Publishers. 
NAHAPIET, J. y GHOSHAL, S. (1998). Social capital, intellectual capital and the organizational advantage. Academy of Management Review, 23 (2), 242-266.

NELSON, R. (ed.) (1993). National Innovation Systems: A Comparative Analysis. Oxford, Oxford University Press.

NELSON, R. y ROSENBERG, N. (1993). Technical innovation and national systems, en: Nelson, R. (ed.). National Innovation Systems: A comparative Analysis. New York: Oxford University Press.

NONAKA, I., SASAKI, K. y AHMED, M. (2003). Continuous innovation: The power of tacit knowledge. En: L. V. Shavinina (Ed.), International Handbook on Innovation. Oxford: Elsevier Science.

OECD (2005). Oslo Manual, $3^{\text {a }}$ edición. Centre Francais d'explotation, Paris (Francia). Disponible en: http://www.oecd.org

OLAZARÁN, M. y GÓMEZ, M. (eds.) (2000). Sistemas Regionales de Innovación. Servicio Editorial de la Universidad del País Vasco.

PAVITT K. (2003). The Process of Innovation. Science and Technology Policy Research -SPRU-. University of Sussex: The Freeman Center. Disponible en: http://www.sussex.ac.uk/Units/spru

ROGERS, E. M. (1995). Diffusion of innovations (4 $4^{\text {th }}$ ed.). New York: The Free Press.

Simulating Self-Organizing innovation networks -SEIN- (1998). Fundada por la Comunidad Económica Europea bajo el programa Socio-Economic Research (TSER), contrato No. SOEI-CT-98-1107.

SWAN, J., SCARBROUGH, H. y Robertson, M. (2003). Linking Knowledge, Networking and Innovation Processes: A Conceptual Model. En: L. V. Shavinina (Ed.), International Handbook on Innovation. Oxford: Elsevier Science.

VÁSQUEZ, Barquero (1999). Desarrollo, redes e innovación: Lecciones sobre desarrollo endógeno. Madrid: Ediciones Pirámide. 